\title{
Selection by Recursively Enumerable Sets ${ }^{\star}$
}

\author{
Wolfgang Merkle ${ }^{1}$, Frank Stephan ${ }^{2}$, Jason Teutsch $^{3}$, Wei Wang ${ }^{4}$, Yue Yang ${ }^{2}$ \\ ${ }^{1}$ Institut für Informatik, Universität Heidelberg, 69120 Heidelberg, Germany. \\ merkle@math.uni-heidelberg.de \\ 2 Department of Mathematics, National University of Singapore, \\ Singapore 119076, Republic of Singapore. \\ fstephan@comp.nus.edu.sg and matyangy@nus.edu.sg \\ ${ }^{3}$ Computer Science and Engineering, Pennsylvania State University. \\ teutsch@cse.psu.edu \\ ${ }^{4}$ Department of Philosophy, Sun Yat-Sen University, \\ 135 Xingang Xi Road, Guangzhou 510275, Guangdong, P.R. of China. \\ wwang.cn@gmail . com
}

\begin{abstract}
For given sets $A, B$, and $Z$ of natural numbers where the members of $Z$ are $z_{0}, z_{1}, \ldots$ in ascending order, one says that $A$ is selected from $B$ by $Z$ if $A(i)=B\left(z_{i}\right)$ for all $i$. Furthermore, say that $A$ is selected from $B$ if $A$ is selected from $B$ by some recursively enumerable set, and that $A$ is selected from $B$ in $n$ steps iff there are sets $E_{0}, E_{1}, \ldots, E_{n}$ such that $E_{0}=A, E_{n}=B$, and $E_{i}$ is selected from $E_{i+1}$ for each $i<n$.

The following results on selections are obtained in the present paper. A set is $\omega$-r.e. if and only if it can be selected from a recursive set in finitely many steps if and only if it can be selected from a recursive set in two steps. There is some Martin-Löf random set from which any $\omega$-r.e. set can be selected in at most two steps, whereas no recursive set can be selected from a Martin-Löf random set in one step. Moreover, all sets selected from Chaitin's $\Omega$ in finitely many steps are Martin-Löf random.
\end{abstract}

\section{Introduction}

Post [12] introduced various important reducibilities in recursion theory among which the one-one reducibility is the strictest one; here $A$ is one-one reducible to $B$ iff there is a one-one recursive function $F$ such that $A(x)=B(F(x))$ for all $x$. In a setting of closed left-r.e. sets, Jain, Stephan and Teutsch [2] investigated a strengthening of one-one reductions were it is required in addition that $F$ is strictly increasing or, equivalently, that $F$ is the principal function of an infinite recursive set. The present paper relaxes the latter notion of reducibility and considers reductions given by principal functions of infinite sets that are recursively enumerable (r.e., for short).

Recall that the principal function of an infinite set $Z$ is the strictly increasing function $F$ such that $Z$ can be written as $\{F(0), F(1), \ldots\}$. In case for such $Z$

\footnotetext{
${ }^{\star}$ F. Stephan is supported in part by NUS grant R252-000-420-112. Part of the work was done while W. Merkle, F. Stephan and Y. Yang visited W. Wang at the Sun Yat-Sen University.
} 
and $F$ some set $A$ is reduced to some set $B$ in the sense that $A(x)=B(F(x))$ for all $x$, this reduction could also be viewed as retrieving $A$ from the asymmetric join $B$ where the two "halves" of the join are not coded at the even and odd positions, respectively, of the join as usual but are coded into the positions that correspond to members and nonmembers, respectively, of the set $Z$.

Definition 1. A set $A$ is selected from a set $B$ by a set $Z$ if $Z$ is infinite and for the principal function $F$ of $Z$ it holds that $A(i)=B(F(i))$ for all $i$. A set $A$ is selected from a set $B, A \sqsubset B$ for short, if $A$ is selected from $B$ by some r.e. set. Furthermore, say that $A$ is selected from $B$ in $n$ steps iff there are sets $E_{0}, E_{1}, \ldots, E_{n}$ such that $E_{0}=A, E_{n}=B$ and $E_{i}$ is selected from $E_{i+1}$ for each $i<n$.

The set $B$ has selection rank $n$, if $n$ is the maximum number such that some set $A$ can be selected from $B$ in $n$ steps but not in $n-1$ steps.

It makes sense to consider selection in several steps as the selection relation is not transitive: it follows by Theorems 14 and 15 that there is a Martin-Löf random set from which every recursive set can be selected in two steps but not in one step.

Note that for any infinite set $Z$, the principal function $F$ of $Z$ depends uniquely on $Z$. Furthermore, for a selection of $A$ from $B$ in $n$ steps as in Definition 1 , where $F_{m}$ selects $E_{m}$ from $E_{m+1}$, one can easily see that the function $\widetilde{F}$ given by

$$
\widetilde{F}(y)=F_{n-1}\left(F_{n-2}\left(\ldots F_{2}\left(F_{1}\left(F_{0}(y)\right)\right) \ldots\right)\right)
$$

satisfies that $A(i)=B(\widetilde{F}(i))$. However, since the selection relation is not transitive, in general, the range of $\widetilde{F}$ is not recursively enumerable and one cannot use the function $\widetilde{F}$ to select $A$ from $B$ in one step.

Early research in algorithmic randomness was formulated in terms of admissible selection rules. More precisely, given a certain way of selecting a subsequence from the characteristic sequence of a set, a set is called random iff all of its subsequences selected this way satisfy the condition in the law of large numbers that in the limit the frequencies of the symbols 0 and 1 are both equal to $1 / 2[6]$. Furthermore, van Lambalgen's Theorem [5] states that if one decomposes a set $A$ by selecting along a recursive set and its complement into two infinite halves $B_{0}$ and $B_{1}$ then $A$ is Martin-Löf random iff $B_{0}$ and $B_{1}$ are Martin-Löf random relative to each other. From this viewpoint it is natural to ask whether the choice of the selection along a recursive set can be generalised here to the choice along an r.e. set and how this is compatible with randomness notions. For notions whose definition involves the halting problem, in particular for Kurtz random relative to $K$, Schnorr random relative to $K$ and Martin-Löf random relative to $K$, it can easily be shown that if $B$ has one of these randomness properties and $A$ is selected from $B$ then $A$ has also the same randomness property and that in the case of Martin-Löf randomness relative to $K$, one even gets the full equivalent of van Lambalgen's Theorem.

These initial and obvious connections ask for deeper investigation in order to see how far these correspondences go and thus, one of the central questions 
investigated in this paper is when random sets can be selected from extremely nonrandom ones and vice versa. Furthermore, the notion of $\omega$-r.e. sets - which also play an important role in algorithmic randomness - fits well with the notion of selection by r.e. sets and strong connections are found. Hence, the present work aims at establishing some basic properties of the selection relation and at clarifying its interplay with other established recursion-theoretic notions like Martin-Löf randomness, immunity, enumeration-properties and initial segment complexity.

In the sequel it is shown that a set is $\omega$-r.e. if and only if it can be selected from an infinite and coinfinite recursive set in two steps. Every recursive set $E$ has selection rank of at most 2, where the selection rank is 2 if and only if the set is infinite and coinfinite. Furthermore, the truth-table cylinder of the halting problem has selection rank 1 . Every set selected from $\Omega$ in finitely many steps is Martin-Löf random (but differs from $\Omega$ in case at least one of the selections is nontrivial). There are Martin-Löf random sets which behave differently, for example, all $\omega$-r.e. sets can be selected from some Martin-Löf random set.

\section{Selection and $\omega$-r.e. sets}

Recall that a set $A$ is $\omega$-r.e. iff there is a recursive function $f$ and a sequence of sets $A_{0}, A_{1}, \ldots$ such that the $A_{s}$ form a recursive approximation to $A$ where the number of mind changes is bounded by $f$, that is,

- the sets $A_{s}$ are uniformly recursive, that is, the mapping $(x, s) \mapsto A_{s}(x)$ is a recursive function of two arguments;

- for all $x$ and all sufficiently large stages $s, A(x)=A_{s}(x)$;

- $A_{0}=\emptyset$ and for every $x$ there at most $f(x)$ stages $s$ with $A_{s}(x) \neq A_{s+1}(x)$.

Note that a set $A$ is r.e. if and only if it is $\omega$-r.e. with a bounding function $f$ as above that satisfies the additional constraint that $f(x)=1$ for all $x$. The r.e. sets and $\omega$-r.e. sets have been well-studied in recursion theory $[6,9-11,14]$.

Our first result is that the $\omega$-r.e. sets are closed downwards under the selection relation.

Theorem 2. Assume that $A$ is selected from $B$ and $B$ is an $\omega-$ r.e. set. Then $A$ is an $\omega$-r.e. set, too.

Proof. Let the recursive approximation $B_{0}, B_{1}, \ldots$ and the recursive function $f$ witness that $B$ is an $\omega$-r.e. set. Furthermore, let $W$ be an r.e. set selecting $A$ from $B$. There is a strictly increasing recursive function $g$ such that its range is a recursive subset $W_{0}$ of $W$. Fix some recursive approximation $W_{0}, W_{1}, \ldots$ of $W$ with $W_{0} \subseteq W_{1} \subseteq \ldots$ and let $A_{s}$ be the set selected from $B_{s}$ by $W_{s}$. Then $A_{0}=\emptyset$ because $B_{0}=\emptyset$. Furthermore, it can easily be seen that $A_{s}(n) \neq A_{s+1}(n)$ requires that there is an $x \leq g(n)$ with $W_{s}(x) \neq W_{s+1}(x)$ or $B_{s}(x) \neq B_{s+1}(x)$. Since for each such $x$ these two conditions can be true for at most 1 and for at most $f(x)$ stages $s$, respectively, the total number of stages $s$ where $A_{s}(n) \neq$ $A_{s+1}(n)$ holds is at most $g(n)+1+f(0)+\ldots+f(g(n))$. Hence $A$ is an $\omega$-r.e. set. 
Theorem 3. Let $O$ be the set of odd numbers and $A$ be an $\omega$-r.e. set. Then $A$ can be selected from $O$ in two steps, that is, there is a set $B$ such that $A \sqsubset B$ and $B \sqsubset O$.

Proof. Let the recursive approximation $A_{0}, A_{1}, \ldots$ and the recursive function $f$ witness that $A$ is an $\omega$-r.e. set., and let $h(n)=n^{2} \cdot(1+f(0)+f(1)+\ldots+f(n))$. It suffices to construct $B$ such that, first, $A(n)=B(h(n))$, that is, the range of $h$ selects $A$ from $B$ and, second, the set $B$ is selected from $O$ by some r.e. set $W$.

Split the natural numbers into consecutive intervals $I_{0}, J_{0}, I_{1}, J_{1}, I_{2}, J_{2}, \ldots$ where the length of $I_{n}$ is $1+f(0)+f(1)+\ldots+f(n)$, and the length of $J_{n}$ is $h(0)+1$ for $n=0$ and is $h(n)-h(n-1)$ for $n \geq 1$. Let $W_{0}$ be the union of all $J_{n}$ and let $k_{0}=0$. During stages $s=0,1, \ldots$, one applies the following updates:

1. Let $B_{s}$ be the set selected by $W_{s}$ from $O$;

2. if $A_{s}\left(k_{s}\right) \neq B_{s}\left(h\left(k_{s}\right)\right)$ then let $W_{s+1}=W_{s} \cup\left\{\max \left(I_{k_{s}} \backslash W_{s}\right)\right\}$ else let $W_{s+1}=$ $W_{s}$

3. let $k_{s+1}=\min \left(\left\{k_{s}+1\right\} \cup\left\{j: A_{s}(j) \neq A_{s+1}(j)\right\}\right)$.

Say a stage $s$ is enumerating in case on reaching its second step the condition of the if-clause is satisfied. First it is shown that for every enumerating stage $s$, the set $I_{k_{s}} \backslash W_{s}$ is nonempty, hence indeed the maximum member of this set is enumerated into $W$ during stage $s$. Fix $n$ and consider the enumerations of members of $I_{n}$ into $W$ in step 2. After each such enumeration at some stage $s$, any further such enumeration requires that at some stage $t>s$, one has $k_{t+1} \leq$ $n<k_{t}$, which by step 3 in turn requires that the approximation to $A$ has a mind change of the form $A_{t}(j) \neq A_{t+1}(j)$ where $j \leq n$ and $s<t$. Since for enumerations of distinct members of $I_{n}$ there must be distinct such pairs $(j, t)$, by choice of $f$ at most $1+f(0)+f(1)+\ldots+f(n)=\left|I_{n}\right|$ members of $I_{n}$ are enumerated into $W$.

Next let $z_{0}, z_{1}, \ldots$ be the members of $W$ in ascending order and for all $s$ let $z_{0}^{s}, z_{1}^{s}, \ldots$ be the members of $W_{s}$ in ascending order. By choice of the lengths of the intervals $J_{n}$ and since $W_{0}$ was chosen as the union of these intervals, one has $z_{h(n)}^{0}=\max J_{n}$ for all $n$. Furthermore, at most $\left|I_{0}\right|+\cdots+\left|I_{n}\right| \leq\left|J_{n}\right|$ times at some stage $s$ a number smaller than $z_{h(n)}^{s}$ is enumerated into $W_{s+1}$. Hence for all such stages, one has

$$
z_{h(n)-1}^{s}=z_{h(n)}^{s}-1 \text {, hence } z_{h(n)}^{s+1}=z_{h(n)}^{s}-1 \text { and } O\left(z_{h(n)}^{s+1}\right)=1-O\left(z_{h(n)}^{s}\right) .
$$

In particular, during each enumerating stage $s$ the value of the previous approximation to $B\left(h\left(k_{s}\right)\right)$ is flipped from $O\left(z_{h\left(k_{s}\right)}^{s}\right)$ to $O\left(z_{h\left(k_{s}\right)}^{s+1}\right)$, and after step 2 of each stage $s$, one has $A_{s}\left(k_{s}\right)=B_{s+1}\left(h\left(k_{s}\right)\right)$. By induction on stages one can then show as an invariant of the construction that during each stage $s$ at the end of step 2 it holds that

$$
A_{s}(j)=B_{s+1}(h(j))=O\left(z_{h(j)}^{s+1}\right) \quad \text { for all } s \text { and all } j \leq k_{s} .
$$

This concludes the verification of the construction because $k_{s}$ goes to infinity by step 3 and because the sets $A_{s}, B_{s}$ and $W_{s}$ converge pointwise to $A, B$ and $W$, respectively. 
The two preceding theorems give rise to the following corollary.

Corollary 4. For any set $A$ the following assertions are equivalent:

1. A is w-r.e.;

2. A can be selected from $O$ in two steps;

3. A can be selected from $O$ in finitely many steps.

As a further consequence of Theorem 3, there is a set $B$ that is selected from a recursive set but has a logarithmic lower bound on the plain Kolmogorov complexity $C(\sigma)$ of its initial segments $\sigma=B(0) B(1) \ldots B(n)$, hence, in particular, the set $B$ is complex [3]. Here the plain Kolmogorov complexity $C(\sigma)$ of a string $\sigma$ is the length of the shortest program $p$ such that $U(p)=\sigma$ for some fixed underlying universal machine $U$, see the textbook of Li and Vitányi [6] for more details. Note that the bound in Corollary 5 is optimal up to a constant factor by the proof of Theorem 11 below, which yields as a special case that every set selected from a recursive set has infinitely many initial segments of at most logarithmic complexity.

Corollary 5. There is a set $B$ selected from $O$ such that for almost all $n$ it holds that $C(B(0) B(1) \ldots B(n)) \geq 0.5 \cdot \log (n)$.

Proof. Section 3 provides a closer look at Chaitin's $\Omega$, which is the standard example of a Martin-Löf random left-r.e. set. From these properties it is immediate that for almost all $n$ it holds that $C(\Omega(0) \Omega(1) \ldots \Omega(n))>n-3 \log n$ and that $\Omega$ is $\omega$-r.e. with bounding function $f(n)=2^{n+1}-1$. Applying the construction in the proof of Theorem 3 with $A$ equal to $\Omega$, one has $1+f(0)+f(1)+\ldots+f(n) \leq 2^{n+2}$, hence $h(n) \leq 3^{n}$ for almost all $n$. So one can retrieve $\Omega(0) \Omega(1) \ldots \Omega(n)$ from $B(0) B(1) \ldots B\left(3^{n}\right)$. The corollary now follows by some elementary rearrangements.

Proposition 7 determines the rank of certain sets with rank 0,1 or 2 . The corresponding arguments use again Theorems 3 and 11, together with the following absorption principle for selections by recursive sets.

Proposition 6. Let $A$ be selected from $E$ by the r.e. set $W$ and let $E$ be selected from $B$ by the recursive set $V$. Then $A$ is selected from $B$.

Proof. Let $v_{0}, v_{1}, \ldots$ and $w_{0}, w_{1}, \ldots$ be the members of $V$ and $W$, respectively, in ascending order. Note that $n \in A$ iff $w_{n} \in E$ iff $v_{w_{n}} \in B$, hence $A$ is selected from $B$ by the r.e. set $\left\{v_{w_{0}}, v_{w_{1}}, \ldots\right\}$.

Proposition 7. Exactly the sets $\emptyset$ and $\mathbb{N}$ have selection rank 0 . Every finite and every cofinite set that differs from $\emptyset$ and $\mathbb{N}$ has selection rank 1 . Every recursive set that is infinite and coinfinite has selection rank 2 .

Proof. The only set that can be selected from the empty set is the empty set itself, hence the empty set has rank 0 ; the same argument works for $\mathbb{N}$. Next consider a finite set $B$. In case $B$ differs from $\emptyset$ and $\mathbb{N}$, some set different from $B$ 
can be selected from $A$, thus the selection rank of $B$ is at least 1 . However, in case a set $A$ is selected from $B$ in several steps, then $A$ and all the intermediate sets are finite and all selecting sets can be taken to be recursive. Then $B$ can be selected from $A$ in a single step according to Proposition 6 , hence the selection rank of $A$ is at most 1. Again, an almost identical argument works for the symmetric case of a coinfinite set.

The selection rank of $O$ is at most 2 by Corollary 2, and is at least 2 because by Theorems 3 and 11 the $\omega$-r.e set $\Omega$ can be selected from $O$ in two steps but not in one step. Given any infinite and coinfinite recursive set $B$, the set $B$ can be selected from $O$ by a recursive set and vice versa. By the absorption principle in Proposition 6, from $B$ and $O$ exactly the same sets can be selected in exactly the same number of steps, hence $B$ and $O$ share the same selection rank.

Proposition 8 shows that also nonrecursive sets can have a low selection rank. The proof of the proposition is based on the fact that every set weakly truth-table reducible to the halting problem $\mathrm{K}$ is also one-one reducible to its truth-table cylinder by a strictly increasing reduction function; however, due to lack of space, details are omitted. Recall that by definition $A$ is truth-table reducible to $B$ if there are recursive functions $f$ and $g$ where $f$ maps pairs of numbers and strings to bits and the reduction is given by $A(x)=f(x, B(0), B(1), \ldots, B(g(x)))$ for every $x$. Recall further that by definition a set $B$ is a truth-table cylinder if there are three recursive functions pad, and, neg such that for all $x$ and $y, \operatorname{pad}(x)>x$, $B(\operatorname{pad}(x))=B(x), B(\operatorname{neg}(x))=1-B(x)$ and $B(\operatorname{and}(x, y))=B(x) \cdot B(y)$. Furthermore, for any set $X$, one can choose a truth-table cylinder in the truthtable degree of $X$ and by appropriately restricting this choice to a specific truthtable cylinder obtain the truth-table cylinder $X^{\mathrm{tt}}$ of $X$.

Proposition 8. The truth-table cylinder $\mathrm{K}^{\mathrm{tt}}$ of the halting problem has selection rank 1 and every $\omega$-r.e. set can be selected from it by a recursive set $W$.

\section{Selection and $\Omega$}

Chaitin's $\Omega$ is a standard example for a Martin-Löf random set which is in addition also an $\omega$-r.e. set [1]. It will turn out that $\Omega$ has various special properties and some but not all of them are shared by Martin-Löf random sets in general. The following gives an overview about Martin-Löf randomness.

Using a characterisation of Schnorr [13], one can say that a set $A$ is MartinLöf random [7] iff no r.e. martingale $M$ succeeds on $A$. In this context, a martingale is a function from binary strings to nonnegative real numbers such that $M(\sigma)=(M(\sigma 0)+M(\sigma 1)) / 2 . M$ succeeds on a set $A$ iff the set $\{M(\sigma): \sigma \preceq A\}$ has the supremum $\infty$, where $\sigma \preceq A$ means that $\sigma(x)=A(x)$ for all $x$ in the domain of $\sigma$; similarly one can compare strings with respect to $\preceq$. Furthermore, $M$ is called r.e. iff $\left\{(\sigma, q): \sigma \in\{0,1\}^{*}, q \in \mathbb{Q}, M(\sigma)>q\right\}$ is recursively enumerable; $M$ is recursive iff the just defined set is recursive. Without loss of generality, one can take a recursive martingale to be $\mathbb{Q}$-valued and can show that whenever some recursive martingale succeeds on $A$ then some $\mathbb{Q}$-valued 
recursive martingale succeeds on $A$ where in addition the function $\sigma \mapsto M(\sigma)$ is a recursive mapping which returns on input $\sigma$ the canonical representation (as a pair of numerator and denominator) of $M(\sigma)$. This also holds relativised to oracles. Furthermore, one can say that a partial-recursive martingale $M$ succeeds on $A$ iff for every $\sigma \preceq A, M(\sigma), M(\sigma 0), M(\sigma 1)$ are all defined, for every $\sigma \preceq A$ the relation $M(\sigma)=(M(\sigma 0)+M(\sigma 1)) / 2$ holds and the supremum of $\{M(\sigma): \sigma \preceq A\}$ is $\infty$. It is known that if a recursive martingale succeeds on $A$, then also a partial-recursive martingale succeeds on $A$; furthermore, if a partial-recursive martingale succeeds on $A$ then a r.e. martingale succeeds on $A$. A further characterisation by Zvonkin and Levin [15] is that $A$ is Martin-Löf random iff there is no partial-recursive function $G$ compressing $A$. Here $G$ compresses $A$ iff $G$ maps strings to strings, the domain of $G$ is prefix free - that is whenever $G(p)$ is defined then $G(p q)$ is undefined for all $p, q \in\{0,1\}^{*}$ with $q \neq \varepsilon-$ and there are infinitely many $n$ for which there is a $p$ of length at most $n$ with $G(p)=A(0) A(1) \ldots A(n)$. The interested reader is referred to standard textbooks on algorithmic randomness for more information $[6,9]$.

In this section, the relations between $\Omega$ and $\sqsubset$ are investigated. First, Theorem 9 shows every set selected from $\Omega$ is Martin-Löf random. Second, the next result shows that one cannot select $\Omega$ nontrivially in several steps from itself, that is, there are no sets $E_{0}, E_{1}, \ldots, E_{n}$ such that $E_{m} \sqsubset E_{m+1}$ via an $W_{e_{m}} \neq \mathbb{N}$ for all $m<n$ and $E_{0}=E_{n}=\Omega$.

Note that for this section, for an r.e. set $W$ with recursive enumeration $W_{0}, W_{1}, \ldots$ (that is, the $W_{s}$ are uniformly recursive, $W=\bigcup_{s} W_{s}$ and $W_{0} \subseteq$ $\left.W_{1} \subseteq \ldots\right)$, one defines the convergence module $c_{W}(x)$ is the first stage $s \geq x$ such that $W_{s}(y)=W(y)$ for all $y \leq x$. Furthermore, one fixes an approximation $\Omega_{0}, \Omega_{1}, \ldots$ from the left for $\Omega$, that is, this approximation satisfies the following three conditions:

- the $\Omega_{s}$ are uniformly recursive;

- for all $x$ and almost all $s, \Omega(x)=\Omega_{s}(x)$;

- whenever $\Omega_{s+1} \neq \Omega_{s}$, then the least element $x$ in the symmetric difference satisfies $x \in \Omega_{s+1}-\Omega_{s}$.

Now one defines the convergence module of $\Omega$ at $x$ as $c_{\Omega}(x)=\min \{s \geq x: \forall y \leq$ $\left.x\left[\Omega_{s}(y)=\Omega(y)\right]\right\}$. Note that $c_{\Omega}$, due to $\Omega$ being Martin-Löf random, grows much faster than $c_{W}$ for any given r.e. set $W$; in particular there is a constant $c$ such that, for all $x>0, c_{\Omega}(x-1)+c \geq c_{W}(x)$. This is used in several of the proofs below, in particular as martingales working on $\Omega$ and currently having the task to predict $\Omega(x)$, can from the already known values $\Omega(0) \ldots \Omega(x-1)$ figure out which $y \leq x$ are in finitely many fixed r.e. sets and therefore reconstruct the nature of reductions up to $x$.

Theorem 9 answers an open question by Kjos-Hanssen, Stephan and Teutsch [4, Question 6.1] on whether a set selected from $\Omega$ by an r.e. set is Martin-Löf random; the corresponding question with respect to selections by co-r.e. sets also mentioned there is still open.

Theorem 9. If $A$ is selected from $\Omega$ in finitely many steps then $A$ is Martin-Löf random. 
Proof. Assume that there are a number $n$ and sets $E_{0}, E_{1}, \ldots, E_{n}, A=E_{0}$, $\Omega=E_{n}$ and for all $m<n$ there is an increasing function $F_{m}$ with $x \in E_{m} \Leftrightarrow$ $F(x) \in E_{m+1}$ and the range of $F_{m}$ being an r.e. set. Note that one knows $F_{m}(0), F_{m}(1), \ldots, F_{m}(y)$ at time $s$ iff all elements of the range of $F_{m}$ below $F_{m}(y)$ are enumerated within $s$ time steps (with respect to some given recursive enumeration of the range of $F_{m}$ ). As the convergence module of $\Omega$ dominates the convergence module of every r.e. set there is a constant $c$ such that one can, for every $x>0$ and every $m<n$, compute $F_{m}(y)$ for all $y$ with $F_{m}(y) \leq x$ within time $c_{\Omega}(x-1)+c$.

Now assume by way of contradiction that $A$ is not Martin-Löf random. Miller [8] showed that there is an oracle $B$ which is low for $\Omega$ and PA-complete; that is, $B$ satisfies that $\Omega$ is Martin-Löf random relative to $B$ and that every partialrecursive $\{0,1\}$-valued function has a total $B$-recursive extension. It is known that every set which is not Martin-Löf random is not recursively random relative to such an oracle $B$; hence there is a $B$-recursive martingale $M$ which succeeds on $A$.

Now it is shown that $M$ can be transformed into a partial $B$-recursive martingale $N$ succeeding on $\Omega$ in contradiction to the choice of $B$; this $N$ will be defined inductively and the $N(\sigma a)$ will be defined for all $\sigma \preceq \Omega$ and all $a \in\{0,1\}$. This is done by inductively defining sequences $\Phi(\sigma)$ from $\sigma$ for some partial-recursive function $\Phi$ and then letting $N(\sigma)=M(\Phi(\sigma))$. As a starting point, let $\Phi(\varepsilon)=\varepsilon$ and hence $N(\varepsilon)=M(\varepsilon)$. Inductively, $\Phi(\sigma a)$ is defined from $\Phi(\sigma)$ and hence $N(\sigma a)$ from $N(\sigma)$.

Now for any given $\sigma$ where $\Phi(\sigma)$ and $N(\sigma)$ are defined, one does for $a=0,1$ the following: Let $s=t+c$ for the first time $t \geq|\sigma|$ with $\sigma \preceq \Omega_{t}$; if this time $t$ does not exist then $N(\sigma 0)$ and $N(\sigma 1)$ are undefined. Having $s$, one computes approximations $F_{m, 0}, F_{m, 1}, \ldots$ to $F_{m}$ where $F_{m, s}(y)$ is the $y$-th element of the set of strings enumerated into the range of $F_{m}$ within $s$ steps with respect to some recursive enumeration. Let

$$
\widetilde{F}_{s}(y)=F_{n-1, s}\left(F_{n-2, s}\left(\ldots\left(F_{1, s}\left(F_{0, s}(y)\right)\right) \ldots\right)\right)
$$

and $\widetilde{F}(y)$ be the limit of $\widetilde{F}_{s}(y)$. Note that when $\sigma \preceq \Omega$ then $\widetilde{F}_{s}(y)=\widetilde{F}(y)$ for all $y$ with $\widetilde{F}(y) \leq|\sigma|+1$ because of the above domination properties; note that the $t$ there would be $c_{\Omega}(|\sigma|)$. Furthermore, for all $y$, either $\widetilde{F}_{s}(y)$ is undefined or $\widetilde{F}_{s}(y) \geq \widetilde{F}(y)$.

If there is a $y$ such that $\widetilde{F}_{s}(y)=|\sigma|$ then let $\Phi(\sigma a)=\Phi(\sigma) a$ else let $\Phi(\sigma a)=\Phi(\sigma)$. Furthermore, $N(\sigma a)=M(\Phi(\sigma a))$.

Now one analyses the behaviour of $N$ on $\Omega$. Note that whenever $\sigma a \preceq \Omega$ and $\widetilde{F}_{s}(y) \in \operatorname{dom}(\sigma a)$ then $\widetilde{F}_{s}(y)=\widetilde{F}(y)$ where the $s$ is as above. As a consequence, one has for the maximal $y$ with $\widetilde{F}_{s}(y) \in \operatorname{dom}(\sigma a)$ that $\Phi(\sigma a)=$ $\Omega(\widetilde{F}(0)) \Omega(\widetilde{F}(1)) \ldots \Omega(\widetilde{F}(y))$ and hence $\Phi(\sigma a) \preceq A$. It follows that $N$ works on $\Omega$ like a delayed version of $M$ on $A$; in particular as $M$ takes on $A$ arbitrarily large values, so does $N$ on $\Omega$. This would mean that $N$ succeeds on $\Omega$ in contradiction to the assumption that $\Omega$ is Martin-Löf random relative to the oracle $B$. Thus, against the assumption, the set $A$ has to be Martin-Löf random. 
A similar proof (which is omitted due to page constraints) shows the following result.

Theorem 10. One cannot select $\Omega$ nontrivially in several steps from itself, that is, there are no $n>0$ and no sets $E_{0}, E_{1}, \ldots, E_{n}$ such that $E_{m} \sqsubset E_{m+1}$ via an $W_{e_{m}} \neq \mathbb{N}$ for all $m<n$ and $E_{0}=E_{n}=\Omega$.

Furthermore, one can also show the following: interchange even and odd positions by letting $\widetilde{\Omega}(2 n)=\Omega(2 n+1)$ and $\widetilde{\Omega}(2 n+1)=\Omega(2 n)$; the set $\widetilde{\Omega}$ cannot be selected from $\Omega$ in any number of steps.

\section{Selection and Martin-Löf random sets in general}

After having investigated relations between selection and the special MartinLöf random set $\Omega$, the focus is now on relations between selection and MartinLöf random sets in general. First, Theorems 11 and 12 exhibit classes of sets from which no Martin-Löf random set can be selected in one step. Furthermore, Theorems 14 and 15 assert that there is a Martin-Löf random set from which one can select all $\omega$-r.e. sets in up to two steps, whereas no recursive set can be selected from any Martin-Löf random set in one step.

Theorem 11. Assume that $B$ is Turing reducible to a Turing-incomplete r.e. set. Then no set selected from $B$ is Martin-Löf random.

Proof. Let $B$ be stated as in the theorem. Recall that a sufficient criterion for a set $A$ to be not Martin-Löf random is that there are infinitely many $n$ such that the plain Kolmogorov complexity of $A(0) A(1) \ldots A(n)$ is bounded proportionally to $\log (n)$. Indeed, in the following it is shown that there are a constant $c$ and infinitely many $n$ such that $C(A(0) A(1) \ldots A(n)) \leq 2 \cdot \log (n)+c$.

Consider any $A \sqsubset B$ and let $W$ be the r.e. set with $A(n)=B\left(w_{n}\right)$ for the $n$-th element $w_{n}$ of $W$ in ascending order. Let $b_{0}, b_{1}, b_{2}, \ldots$ be a recursive one-one enumeration of $W$ and let $e_{0}=0$ and $e_{n+1}$ be the first number $d>e_{n}$ such that $b_{e_{n}}<b_{d}$. Note that the mapping $m \mapsto b_{e_{m}}$ is recursive. Now given any $m$, let $n$ be the number with $b_{e_{m}}=w_{n}$, note that $m \leq n$. Knowing $m$ and $n$, one can compute $w_{0}, w_{1}, \ldots, w_{n}$.

There is a recursive approximation $B_{0}, B_{1}, \ldots$ to $B$ such that the convergence module $g$ of this approximation does not permit to compute the diagonal halting problem $K$. In particular there are infinitely many $m \in K$ such that $m$ is enumerated into $K$ at a stage $s$ larger than $g\left(b_{e_{m}}\right)$ and all $w_{k}$ with $k \leq n$ satisfy $B_{s}\left(w_{k}\right)=B\left(w_{k}\right)$. Hence, for these $m$ and the corresponding $n, A(0) A(1) \ldots A(n)$ can be described by $m$ and $n$ using the time $s$ when $m$ is enumerated into $K$ and the members $w_{0}, w_{1}, \ldots, w_{n}$ of $W$ obtained from $m, n$ and conjecturing that $A(k)=B_{s}\left(w_{k}\right)$ for $k=0,1, \ldots, n$. For the right parameters, the $s$ exists and the corresponding data can be computed and the resulting string is correct. As one can describe $m$ and $n$ by two numbers of $\log (n)$ binary digits (the number of digits must be the same for permitting to separate out the digits from $m$ from those for $n), C(A(0) A(1) \ldots A(n)) \leq 2 \cdot \log (n)+c$ for some constant $c$ and infinitely many $n$. It follows that $A$ is not Martin-Löf random. 
Theorem 12. Every truth-table degree contains a set B such that no set selected from $B$ is Martin-Löf random.

Proof. This proof is mainly based on the fact that every truth-table degree contains a retraceable set; here a set $B$ is retraceable iff there is a partial-recursive function $\psi$ which returns for every $x \in B$ a canonical index of the set $\{y \leq x: y \in$ $B\}$; on $x \notin B, \psi$ can either be undefined or return any information, either wrong or right. For example, if $E$ is a given set then the set $B=\left\{x_{0}, x_{1}, \ldots\right\}$ with $x_{0}=1$ and $x_{n+1}=2 x_{n}+E(n)$ for all $n$ is a retraceable set of the same truth-table degree as $E$. So fix such $B$ and $\psi$ with $B$ being inside the given truth-table degree. The proof follows now in general the proof of Theorem 11 with the adjustment that it is shown that for each $A \sqsubset B$ there are a constant $c$ and infinitely many $n$ such that the plain Kolmogorov complexity of $A(0) A(1) \ldots A(n)$ is bounded by $3 \cdot \log (n)+c$, which then gives that $A$ is not Martin-Löf random.

Consider any $A \sqsubset B$ and let $W$ be the r.e. set with $A(n)=B\left(w_{n}\right)$ for the $n$-th element $w_{n}$ of $W$ in ascending order. Without loss of generality, $0 \in A$. Let $b_{0}, b_{1}, b_{2}, \ldots$ be a recursive one-one enumeration of $W$ and let $e_{0}=0$ and $e_{n+1}$ be the first number $d>e_{n}$ such that $b_{e_{n}}<b_{d}$. Note that the mapping $m \mapsto b_{e_{m}}$ is recursive. Now given any $m$, let $n$ be the number with $b_{e_{m}}=w_{n}$, note that $m \leq n$. Knowing $m$ and $n$, one can compute $w_{0}, w_{1}, \ldots, w_{n}$. Furthermore, let $k$ be such that $w_{k}$ is the maximal of the $w_{0}, w_{1}, w_{2}, \ldots, w_{n}$ with $w_{k} \in B$. Note that $k \leq n$ and $k$ exists as $0 \in A \wedge w_{0} \in B$.

Hence, for each $n$ and the corresponding $m, k \leq n$, one can compute $w_{0}, w_{1}$, $\ldots, w_{n}$ from $m, n$ and use $\psi\left(w_{k}\right)$ to find out which of these numbers are in $B$. Hence $A(0) A(1) \ldots A(n)$ can be computed from $n, m, k$. One can code $m, n, k$ as 3 binary numbers of $\log (n)$ digits each and gets therefore that there are a constant $c$ and infinitely many $n$ such that $C(A(0) A(1) \ldots A(n)) \leq 3 \cdot \log (n)+c$. Hence the set $A$ is not Martin-Löf random.

If one would start with a hyperimmune set $B$ then every $A \sqsubset B$ is also hyperimmune and therefore not Martin-Löf random. Hence one has the following result similar to the previous one.

Proposition 13. There are uncountably many sets $B$ such that no set that is selected from $B$ in one or several steps is Martin-Löf random.

The following result stands in contrast to Theorem 9, which says that one cannot select any nonrandom set from $\Omega$ in arbitrarily many steps. Note that the resulting set $B$ is like $\Omega$ also an $\omega$-r.e. Martin-Löf random set. The lengthy proof is omitted due to page constraints.

Theorem 14. There is a Martin-Löf random set B such that some set selected from $B$ is not Martin-Löf random and every $\omega$-r.e. set can be selected from $B$ in two steps.

Theorem 15 below shows that the above bound of two steps cannot be brought down to one; indeed, recursive sets can be selected from the above $B$ in exactly two steps. Note that the proof of Theorem 15 indeed shows that it is not possible 
to select from a Martin-Löf random set any set that obeys certain upper bounds on the complexity of its initial segments.

Theorem 15. It is not possible to select a recursive set from a Martin-Löf random set.

Proof. Assume that $A \sqsubset B$ via an r.e. set $W$ and $A$ is recursive and $F$ is the function which lists $W$ in ascending order ( $F$ is not recursive). So $A(x)=B(F(x))$ for all $x$ and $W=\{F(0), F(1), \ldots\}$. Let $u_{0}, u_{1}, \ldots$ be an ascending recursive enumeration of a recursive subset of $W$ which is selected such that $W$ has at least $3^{n}$ elements below a given $u_{n}$. Now one shows that there is a partial-recursive function $G$ with prefix-free domain which compresses $B$, that is, for which there are infinitely many $p \in \operatorname{dom}(G)$ with $G(p)$ being a prefix of $B$ which is longer than $|p|$; this would then be an alternative way to prove that $B$ is not Martin-Löf random.

On input $p=0^{n} 10^{m} 1 b_{0} b_{1} \ldots b_{m} c_{0} c_{1} \ldots c_{k}, G(p)$ first checks whether $k+1=$ $u_{n}-d$ where $d$ is the binary value of $b_{0} b_{1} \ldots b_{m}$. In the case that this is true, $G(p)$ enumerates the $W$ until $d$ many elements at places $\widetilde{F}(0), \widetilde{F}(1), \ldots, \widetilde{F}(d)$ have been enumerated into $W$ with $\widetilde{F}(0)<\widetilde{F}(1)<\ldots<\widetilde{F}(d)=u_{n}$. If this is eventually achieved and if $d \geq n$, then $G$ outputs a string $\sigma \in\{0,1\}^{u_{n}+1}$ which is obtained by letting $\sigma\left(\widetilde{F}\left(d^{\prime}\right)\right)=A\left(d^{\prime}\right)$ for all $d^{\prime} \leq d$ and by filling the remaining missing $k+1$ values in $\sigma$ below the position $u_{n}$ according to the string $c_{0} c_{1} \ldots c_{k}$. This results in a string of length $u_{n}$ which is computed from a $p$ of length $n+2 m+k+4$; by taking $m$ as small as possible, one has that $m \leq \log (d)+1$ and $n \leq \log (d)$, thus one has a length bounded by $u_{n}+3 \log (d)-d$ which is, for all sufficiently large $n$ and $d$ (as $d \geq n$ ) smaller than $u_{n}$.

One has now to show that one can always choose $d, m, b_{0} b_{1} \ldots b_{m}$ and $c_{0} c_{1} \ldots c_{k}$ such that the corresponding output $G(p)$ is $B(0) B(1) \ldots B\left(u_{n}\right)$. To see this, let $d$ be the number of strings in $W$ up to $u_{n}$ (which is larger than $n)$ and $m=\log (d)$ and $b_{0} b_{1} \ldots b_{m}$ be the binary representation of $d$. Furthermore, let $k=u_{n}-d-1$. One gets that $\widetilde{F}\left(d^{\prime}\right)=F\left(d^{\prime}\right)$ for all $d^{\prime} \leq d$. Now one chooses $c_{0} c_{1} \ldots c_{k}$ such that the missing positions in $\sigma$ which are not covered by $F(0), F(1), \ldots, F(d)$ are covered with the corresponding bits of $B$. Hence one has that for the so selected $p$ that $G(p)$ equals $B(0) B(1) \ldots B\left(u_{n}\right)$. It is furthermore easy to verify that the domain of $G$ is prefix-free.

\section{Conclusion}

The present paper focussed on the question when a set $A$ is one-one reducible to $B$ via the principal function of an r.e. set and generalised this notion also to reductions in several steps, as this reducibility is not transitive. The investigations show that there is a rich relation between this type of reducibility and $\omega$-r.e. sets and Martin-Löf random sets. Future work might in particular address the question for which numbers $n \in\{0,1,2, \ldots, \infty\}$ there are sets $A$ of selection rank $n$; for $n=0,1,2$, examples are given within this paper and all of these 
examples are $\omega$-r.e. sets. As the current investigations centered on $\omega$-r.e. sets, subsequent research might also aim for more insights concerning the selection relation among sets that are not $\omega$-r.e. or even not $\Delta_{2}^{0}$. For example, one might ask whether every set selected from a strongly random set in finitely many steps is again strongly random; this closure property holds for 2-randomness and also for 2-genericity but not for Martin-Löf randomness and also not for 1-genericity.

\section{References}

1. Cristian S. Calude. Chaitin $\Omega$ numbers, Solovay machines and incompleteness. Theoretical Computer Science, 284:269-277, 2002.

2. Sanjay Jain, Frank Stephan and Jason Teutsch. Closed left-r.e. sets. Theory and Models of Computation 2011, Eighth annual conference, TAMC 2011, Tokyo, Japan, May 2011, Proceedings. Springer LNCS 218-229, 2011.

3. Bjørn Kjos-Hanssen, Wolfgang Merkle and Frank Stephan. Kolmogorov complexity and the recursion theorem. Transactions of the American Mathematical Society, 363:5465-5480, 2011.

4. Bjørn Kjos-Hanssen, Frank Stephan and Jason Teutsch. Arithmetic complexity via effective names for random sequences. ACM Transactions on Computational Logic, 13(3):24:1-24:18, 2012.

5. Michiel van Lambalgen. The axiomatization of randomness. The Journal of Symbolic Logic, 55(3):1143-1167, 1990.

6. Ming Li and Paul Vitányi. An Introduction to Kolmogorov Complexity and its Applications. Third Edition. Springer, 2008.

7. Per Martin-Löf, The definition of random sequences. Information and Control, 9:602-619, 1966.

8. Joseph S. Miller. The K-degrees, low for $\mathrm{K}$ degrees, and weakly low for $\mathrm{K}$ sets. Notre Dame Journal of Formal Logic, 50(4):381-391, 2010.

9. André Nies. Computability and Randomness. Volume 51 of Oxford Logic Guides. Oxford University Press, Oxford, 2009.

10. Piergiorgio Odifreddi. Classical Recursion Theory. Studies in Logic and the Foundations of Mathematics, 125. North-Holland, 1989.

11. Piergiorgio Odifreddi. Classical Recursion Theory, Volume II. Studies in Logic and the Foundations of Mathematics, 143. Elsevier, 1999.

12. Emil Leon Post. Recursively enumerable sets of positive integers and their decision problems, Bulletin of the American Mathematical Society, 50:284-316, 1944.

13. Claus Peter Schnorr. Zufälligkeit und Wahrscheinlichkeit. Springer Lecture Notes in Mathematics, 1971.

14. Robert I. Soare. Recursively Enumerable Sets and Degrees. Springer-Verlag, Heidelberg, 1987.

15. Alexander K. Zvonkin and Leonid A. Levin. The complexity of finite objects and the development of the concepts of information and randomness by means of the theory of algorithms. Russian Mathematical Surveys, 25:83-124, 1970. 